\title{
Phytomedical Therapies of Oral Mucositis
}

\author{
Masood Sepehrimanesh* \\ Department of Gastroenterohepatology Research Center, Shiraz University of Medical Sciences, Iran
}

Submission: October 4, 2016; Published: October 7, 2016

*Corresponding author: Masood Sepehrimanesh, Department of Gastroenterohepatology Research Center, Shiraz University of Medical Sciences, Iran, Email: sepehrimaneshmasood@gmail.com

\section{Editorial}

Oral mucositis $(\mathrm{OM})$ is an inflammatory response and adverse effect which routinely occurs in head and neck cancer suffered patients with chemo- and radiotherapy. This can be affected the quality of life due to change in the eating and drinking and also due to its related pain. In OM, first the painful inflammation and ulceration of the mucous membranes lining the oral cavity is occurred and then the lesion may be infected by several pathogenic bacteria. Nowadays, use of traditional medicine such as herbal medicine in treatments of any diseases and disorders are being common due to lower side effects, costs and also appropriate effects.

For instance, I and coworkers reported the beneficial effects of some plant species in diabetes mellitus [1-4], ulcerative colitis [5-8], thyroid abnormal functions [9,10], hyperlipidemia [3], and intestinal morphology [11]. A similar trend is also existed about the OM. Most of clinician's advice to treat this side effect with using of palliative treatments. However, recently the use of herbal medicine in the treatment of $\mathrm{OM}$ is attracting by any scientist. The anti-inflammatory and ant oxidative effects of certain plant species in the treatment of pathological lesion of OM were reported by my team previously $[12,13]$.

It has been cleared that most of plant species and also most parts of each plant contained several effective agents such as polyphenolic compounds and also essential oils which exhibited such therapeutic effects [14-16]. These compounds combat with reactive oxygen species (ROS) and stimulate the healing process in OM. Indeed, the most cause of any lesions in OM is an accumulation of ROS, which influenced the local immune function and deteriorate the healing process by affecting cellular and molecular process.

Conclusively, according to the previous experiences, certain plants exhibited antioxidant properties and also contain some antimicrobial agents. These can be attractive for pharmacologist to purify such constituents and help physicians to apply them as alternative therapies for patient with painful OM lesions. Most published work, include those of my team, and are performed in animal model. Although, their findings are interesting and reliable, but due to interspecies differences, need to human studies are sensed. Therefore, further studies must be conducted in human populations as double blinded randomized clinical trial. I invited young and famous researchers to organize such studies to help cancerous patients for overcoming this painful lesion.

\section{References}

1. Hafezi H, Vahdati A, Sepehrimanesh M (2015) Effect of Satureja khuzestanica Jamzad extract on serum lipid profile, blood glucose level and body weight gain in diabetes mellitus: a Rattus norvegicus model. Comparative Clinical Pathology (24): 1033.

2. Karami M, Sepehrimanesh M, Koohi-Hosseinabadi O, Fattahi M, Razeghian JI, et al. (2016) Therapeutic effects of hydroalcoholic and aqueous extracts of Berberis vulgaris fruits in streptozotocin induced type 1 diabetes mellitus rats. Romanian Journal of Diabetes Nutrition and Metabolic Diseases 23(3): 239-245.

3. Koohi-Hosseinabadi O, Moini M, Safarpoor A, Derakhshanfar A, Sepehrimanesh M (2015) Effects of dietary Thymus vulgaris extract alone or with atorvastatin on the liver, kidney, heart, and brain histopathological features in diabetic and hyperlipidemic male rats. Comparative Clinical Pathology 24(6): 1311-1315.

4. Shojaee SS, Vahdati A, Assaei R, Sepehrimanesh M (2015) Effect of Galega officinalis leaf powder and Trigonella foenum-graecum seed powder on blood glucose levels and weight gain in a diabetes mellitus rat model. Comparative Clinical Pathology. 24(1): 145-148.

5. Hosseinzadeh F, Tanideh N, Azarpira N, Sayarifard A, Sepehrimanesh M, Salehi M (2016) Oral grapeseed oil and sesame oil in experimental acetic acid-induced ulcerative colitis in rat. Annals of Colorectal Research 4(2): e37285.

6. Safarpour AR, Kaviyani F, Sepehrimanesh M, Ahmadi N, Koohi Hosseinabadi O, et al. (2015) Antioxidant and anti-inflammatory effects of gel and aqueous extract of Melilotus officinalis L. in induced ulcerative colitis: A Rattus norvegicus model. Annals of Colorectal Research. 3(2): e29511.

7. Tanideh N, Jamshidzadeh A, Sepehrimanesh M, Hosseinzadeh M, Koohi-Hosseinabadi O, et al. (2016) Healing acceleration of acetic acidinduced colitis by marigold (Calendula officinalis) in male rats. Saudi J Gastroenterol 22(1): 50-56.

8. Tanideh N, Nematollahi SL, Hosseini SV, Hosseinzadeh M, Mehrabani D, et al. (2014) The healing effect of Hypericum perforatum extract 
on acetic acid-induced ulcerative colitis in rat. Annals of Colorectal Research 2(4): e25188.

9. Assaei R, Mostafavi-Pour Z, Pajouhi N, Ranjbar Omrani GH, Sepehrimanesh M, et al. (2015) Effects of essential oil of Satureja khuzestanica on the oxidative stress in experimental hyperthyroid male rat. Vet Res Forum 6(3): 233-238.

10. Nazifi S, Saeb M, Sepehrimanesh M, Poorgonabadi S (2012) The effects of wild pistachio oil on serum leptin, thyroid hormones, and lipid profile in female rats with experimental hypothyroidism. Comparative Clinical Pathology 21(5): 851-857.

11. Yazdani A, Poorbaghi SL, Habibi H, Nazifi S, Sepehrimanesh M et al. (2013) Dietary Berberis vulgaris extract enhances intestinal mucosa morphology in the broiler chicken (Gallus gallus). Comparative Clinical Pathology. 22(4): 611-615.

12. Koohi-Hosseinabadi O, Andisheh-Tadbir A, Bahadori P, Sepehrimanesh M, Mardani M, et al. (2015) Comparison of the therapeutic effects of the dietary and topical forms of Zizyphus jujuba extract on oral mucositis induced by 5-fluorouracil: a golden hamster model. Journal of Clinical and Experimental Dentistry 7(2): e304-e309.

13. Mardani M, Afra SM, Tanideh N, Andisheh Tadbir A, Modarresi F, et al. (2016) Hydroalcoholic extract of Carum carvi L. in oral mucositis: a clinical trial in male golden hamsters. Oral Diseases. 22(1): 39-45.

14. Kazemipour N, Nikbin M, Davarimanesh A, Sepehrimanesh M (2015) Antioxidant activity and mineral element contents of Calotropis procera from Iran: a traditional medicinal plant in Middle East. Comparative Clinical Pathology. 24(5): 1147-1150.

15. Najafian S, Moradi M, Sepehrimanesh M (2016) Polyphenolic contents and antioxidant activities of two medicinal plant species, Mentha piperita and Stevia rebaudiana, cultivated in Iran. Comparative Clinical Pathology 25(4): 743-747.

16. Nikbin M, Kazemipour N, Maghsoodlou MT, Valizadeh J, Sepehrimanesh M, etal. (2014) Mineral elements and essential oil contents of Scutellaria luteo-caerulea Bornm. \& Snit. Avicenna Journal of Phytomedicine 4(3): 182-190.

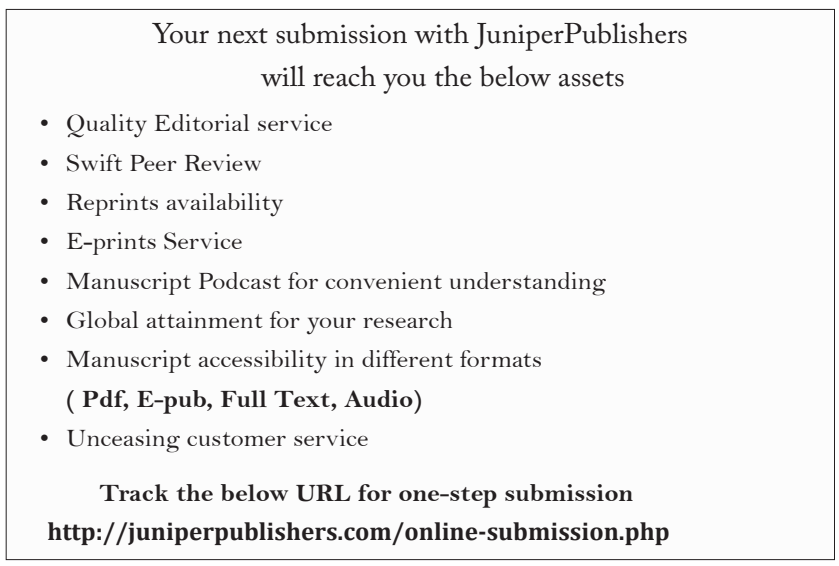

